\title{
Validated method based on immunocapture and liquid chromatography coupled to high-resolution mass spectrometry to eliminate isatuximab interference with M-protein measurement in serum
}

Stéphane Muccio ${ }^{1 *}$, Alexandra Tavernier ${ }^{1}$, Marie-Claude Rouchon ${ }^{1}$, Alain Roccon ${ }^{1}$, Shujia Dai ${ }^{2}$, Greg Finn ${ }^{3}$, Sandrine Macé ${ }^{4}$, Valérie Boutet ${ }^{4}$ and Olivier Fedeli ${ }^{1}$.

${ }^{1}$ Sanofi R\&D, 371 rue du Professeur Blayac, 34184 Montpellier, France ;

2Sanofi Translational Sciences, 640 Memorial Drive, Cambridge, MA 02139, U.S.;

${ }^{3}$ Sanofi Oncology, 640 Memorial Drive, Cambridge MA 02139, U.S.;

${ }^{4}$ Sanofi R\&D, 1 avenue Pierre Brossolette, 91385 Chilly-Mazarin, France.

*Email : stephane.muccio@sanofi.com

Supporting Information: Description of deconvolution process for M-protein LC identification, validation tests results and additional information for specificity, linearity performance, dilution linearity, accuracy and precision, and matrix variability assessments.

Figure S1. Deconvolution process for M-protein LC and isatuximab LC identification using Biopharma Finder software. (A) Base peak chromatogram. (B) Mass spectrum of selected chromatographic peaks. (C) Deconvoluted mass spectrum: flagging of isatuximab LC and identification of M-protein LC.

Figure S2. Extracted ion chromatograms (XIC) for the specificity of the assay. (A) Blank human serum, XIC of alemtuzumab. (B) Blank human serum spiked with alemtuzumab at the LLOQ level $(10 \mu \mathrm{g} / \mathrm{mL}), \mathrm{XIC}$ of alemtuzumab. (C) Blank human serum spiked with alemtuzumab at the LLOQ level $(10 \mu \mathrm{g} / \mathrm{mL})$ and with isatuximab at $100 \mu \mathrm{g} / \mathrm{mL}$, XIC of isatuximab. (D) Blank human serum spiked with alemtuzumab at the LLOQ level $(10 \mu \mathrm{g} / \mathrm{mL})$ and with commercial IgG1 kappa at $100 \mu \mathrm{g} / \mathrm{mL}$, XIC of commercial IgG1 kappa.

Figure S3. Dilution linearity of MS signal for M-protein from 3 patients.

Figure S4. a) Individual M-protein LC retention times (37 patients). b) Individual M-protein LC deconvoluted mass (37 patients).

Table S1. Back-calculated calibration standards for alemtuzumab $(\mu \mathrm{g} / \mathrm{mL})$ in human serum.

Table S2. Calibration curve regression parameters for alemtuzumab.

Table S3. Intra- and inter-runs accuracy and precision of the assay performed using alemtuzumab on three separate occasions.

Table S4. Influence of dilution on the quantification of alemtuzumab $(\mu \mathrm{g} / \mathrm{mL})$ in human serum.

Table S5. Influence of matrix variability on quantitation of commercial M-protein (IgG1 kappa from Sigma at $20.4 \mu \mathrm{g} / \mathrm{mL}$ ) in 6 different batches of serum from healthy individuals (concentrations expressed as $\mu \mathrm{g} / \mathrm{mL}$ alemtuzumab equivalent).

Table S6. M-protein LC deconvoluted mass for the patient with VGPR (Fig. 3) at each treatment cycle showing the high precision and robustness of the method. 


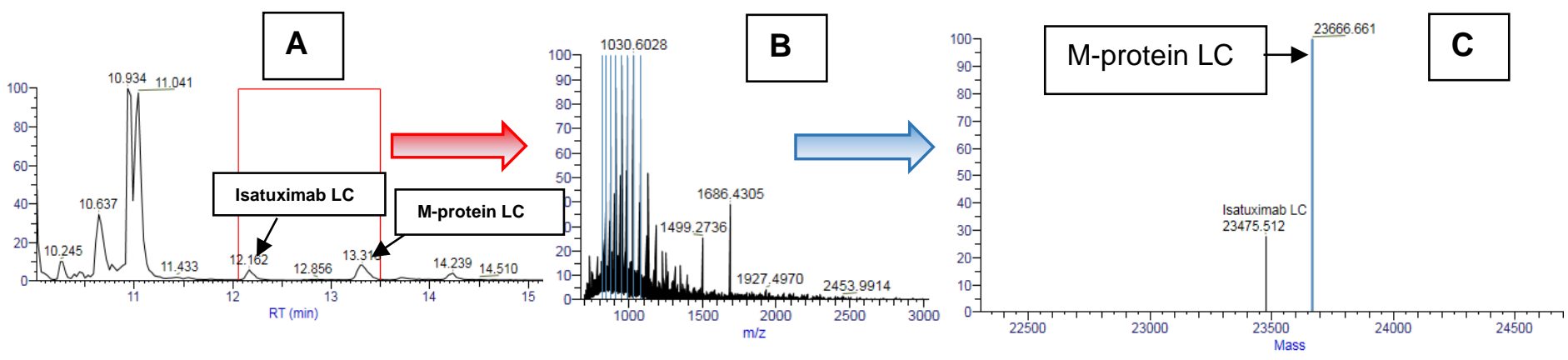

Figure S1. Deconvolution process for M-protein LC and isatuximab LC identification using Biopharma Finder software. (A) Base peak chromatogram. (B) Mass spectrum of selected chromatographic peaks. (C) Deconvoluted mass spectrum: flagging of isatuximab LC and identification of M-protein LC. 

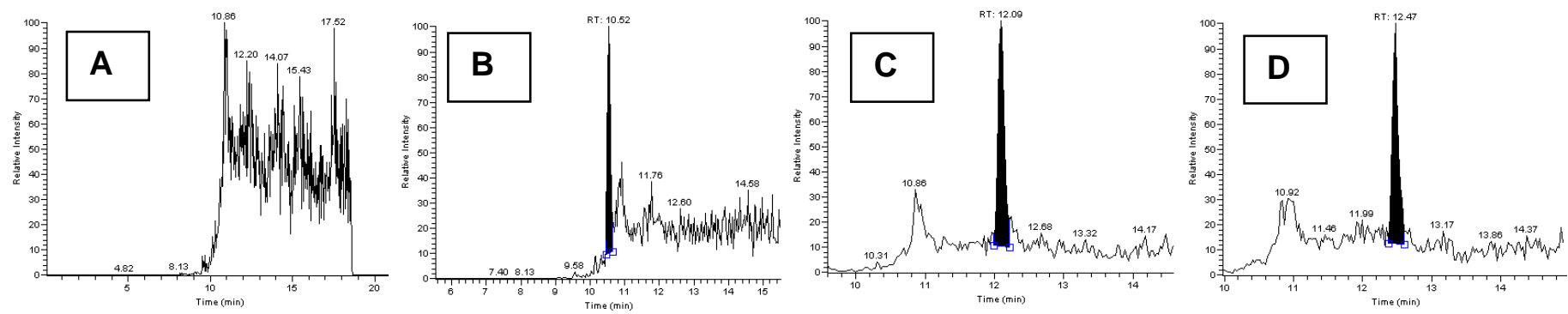

Figure S2. Extracted ion chromatograms (XIC) for the specificity of the assay. (A) Blank human serum, XIC of alemtuzumab. (B) Blank human serum spiked with alemtuzumab at the LLOQ level $(10 \mu \mathrm{g} / \mathrm{mL})$, XIC of alemtuzumab. (C) Blank human serum spiked with alemtuzumab at the LLOQ level $(10 \mu \mathrm{g} / \mathrm{mL})$ and with isatuximab at $100 \mu \mathrm{g} / \mathrm{mL}$, XIC of isatuximab. (D) Blank human serum spiked with alemtuzumab at the LLOQ level (10 $\mu \mathrm{g} / \mathrm{mL})$ and with commercial IgG1 kappa at $100 \mu \mathrm{g} / \mathrm{mL}$, XIC of commercial IgG1 kappa. 

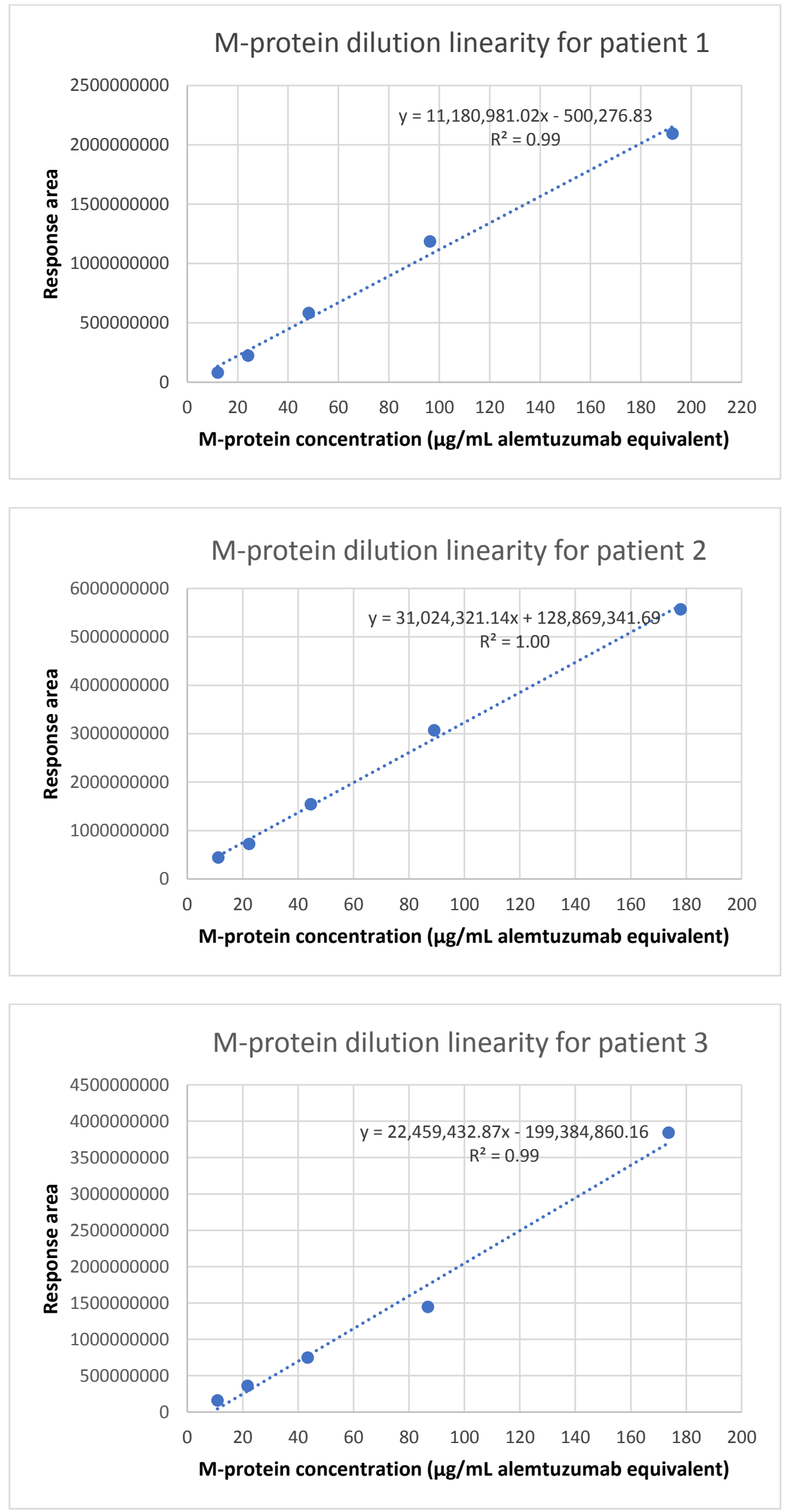

Figure S3. Dilution linearity of MS signal for M-protein from 3 patients. 
a)

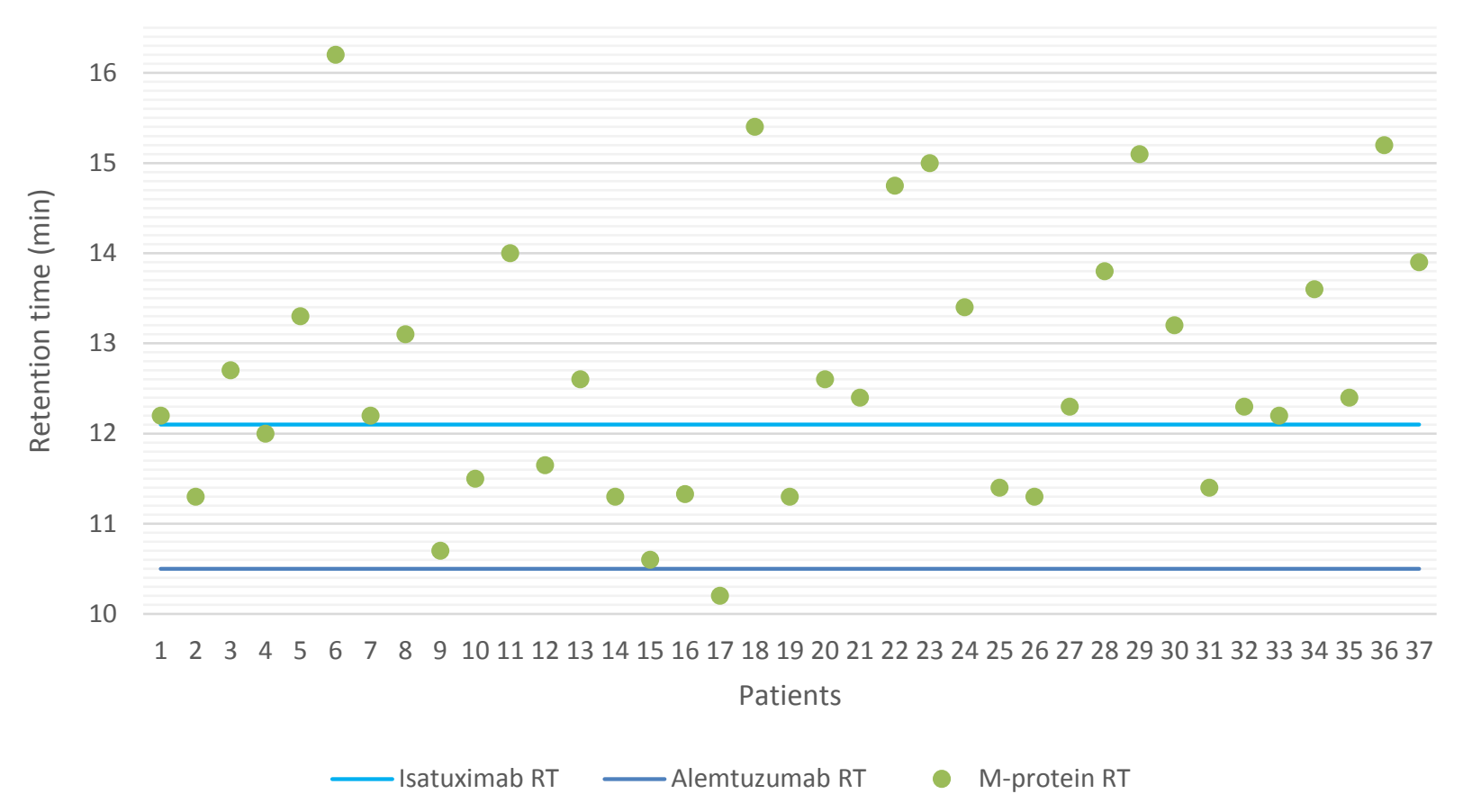

b)

25400

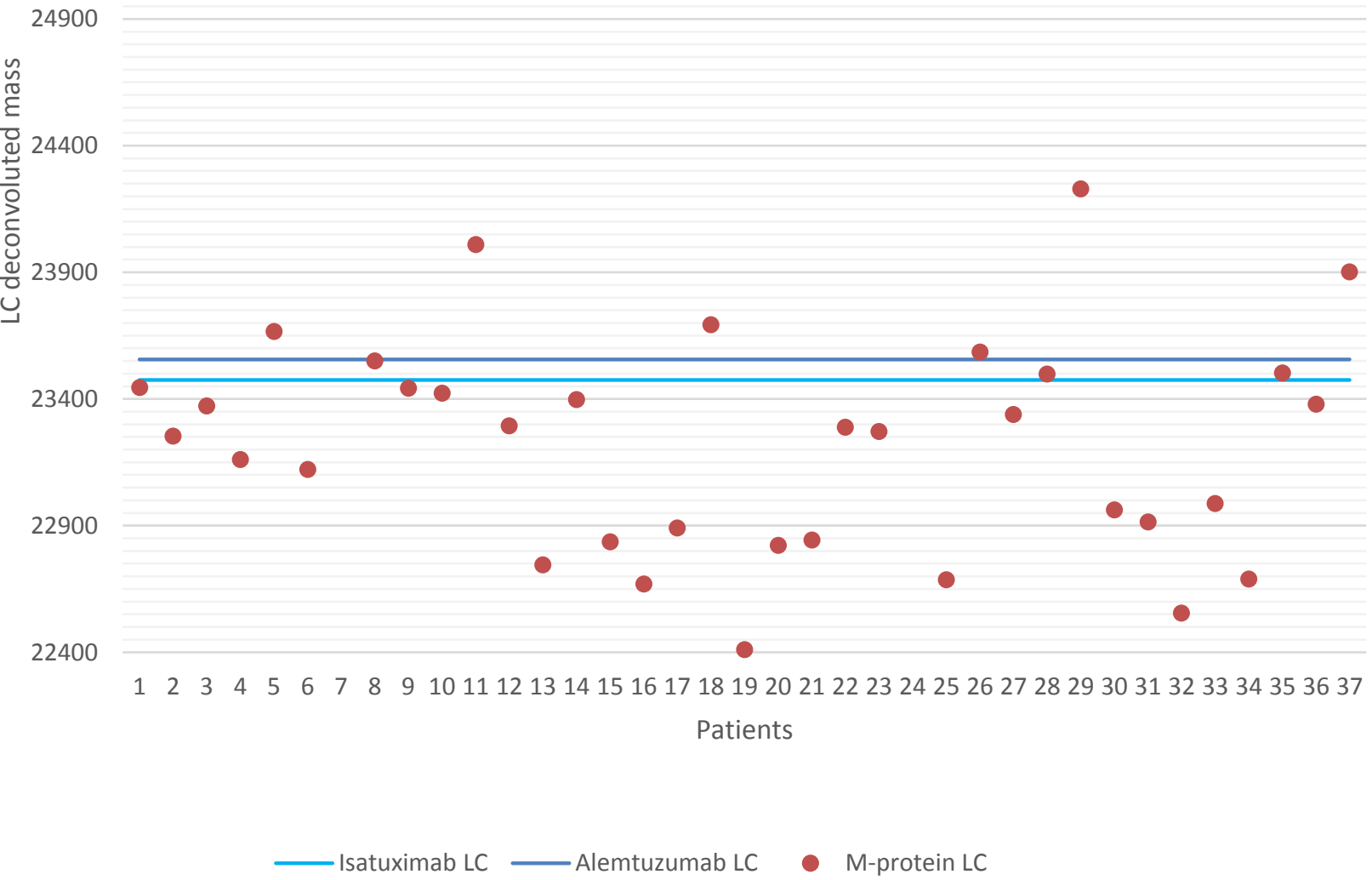

Figure S4. a) Individual M-protein LC retention times (37 patients). b) Individual M-protein LC deconvoluted mass (37 patients). LC masses above $25 \mathrm{kDa}$ correspond to glycosylated forms. 
Table S1. Back-calculated calibration standards for alemtuzumab $(\mu \mathrm{g} / \mathrm{mL})$ in human serum.

\begin{tabular}{|c|c|c|c|c|c|c|c|}
\hline $\begin{array}{c}\text { Analytical Run } \\
\text { Number }\end{array}$ & 10.0 & 20.0 & 50.0 & 75.0 & 100 & 150 & 200 \\
\hline \multirow[t]{3}{*}{1} & 8.67 & 18.2 & 45.2 & 82.9 & 104 & 152 & 191 \\
\hline & 10.3 & & & & & & 191 \\
\hline & 11.5 & & & & & & 210 \\
\hline \multirow[t]{3}{*}{2} & 10.0 & 19.9 & 45.7 & 76.0 & 106 & 153 & 195 \\
\hline & 10.3 & & & & & & 205 \\
\hline & 9.84 & & & & & & 195 \\
\hline \multirow[t]{3}{*}{3} & 10.7 & 18.3 & 47.4 & 75.6 & 110 & 151 & 191 \\
\hline & 9.63 & & & & & & 193 \\
\hline & 10.1 & & & & & & 209 \\
\hline \multirow[t]{3}{*}{4} & 10.8 & 21.3 & 47.8 & 76.1 & 97.4 & 153 & 199 \\
\hline & 9.46 & & & & & & 205 \\
\hline & 9.49 & & & & & & 196 \\
\hline \multirow[t]{3}{*}{5} & 9.77 & 19.4 & 53.7 & 71.5 & 102 & 142 & 215 \\
\hline & 10.2 & & & & & & 188 \\
\hline & 10.1 & & & & & & 203 \\
\hline \multirow[t]{3}{*}{6} & 10.1 & 18.3 & 57.0 & 69.5 & 95.3 & 158 & 203 \\
\hline & 9.98 & & & & & & 186 \\
\hline & 10.2 & & & & & & 208 \\
\hline \multirow[t]{3}{*}{7} & 9.49 & 17.9 & 48.3 & 77.4 & 106 & 152 & 188 \\
\hline & 10.2 & & & & & & 207 \\
\hline & 10.9 & & & & & & 199 \\
\hline \multirow[t]{3}{*}{8} & 9.78 & 19.8 & 48.8 & 74.7 & 104 & 149 & 191 \\
\hline & 9.82 & & & & & & 206 \\
\hline & 10.5 & & & & & & 202 \\
\hline \multirow[t]{3}{*}{9} & 10.0 & 16.6 & 49.9 & 78.9 & 107 & 148 & 184 \\
\hline & 10.2 & & & & & & 210 \\
\hline & 10.5 & & & & & & 201 \\
\hline \multirow[t]{3}{*}{10} & 9.78 & 19.1 & 45.6 & 79.0 & 107 & 145 & 196 \\
\hline & 10.4 & & & & & & 201 \\
\hline & 10.1 & & & & & & 203 \\
\hline \multirow[t]{3}{*}{11} & 9.70 & 18.7 & 45.9 & 77.4 & 104 & 162 & 199 \\
\hline & 10.3 & & & & & & 195 \\
\hline & 10.4 & & & & & & 194 \\
\hline \multirow[t]{3}{*}{12} & 8.31 & 19.1 & 48.9 & 84.6 & 93.2 & 146 & 203 \\
\hline & 11.6 & & & & & & 196 \\
\hline & 10.3 & & & & & & 204 \\
\hline 13 & 8.13 & 22.6 & 43.9 & 85.7 & 90.6 & 145 & 209 \\
\hline
\end{tabular}


Table S1. Back-calculated calibration standards for alemtuzumab $(\mu \mathrm{g} / \mathrm{mL})$ in human serum (continued).

\begin{tabular}{|c|c|c|c|c|c|c|c|}
\hline $\begin{array}{c}\text { Analytical Run } \\
\text { Number }\end{array}$ & 10.0 & 20.0 & 50.0 & 75.0 & 100 & 150 & 200 \\
\hline & 10.2 & & & & & & 197 \\
\hline & 11.1 & & & & & & 200 \\
\hline \multirow[t]{3}{*}{14} & *5.56 & $* * 7.91$ & 48.9 & 74.5 & 107 & 140 & 192 \\
\hline & 10.7 & & & & & & 206 \\
\hline & 9.33 & & & & & & 205 \\
\hline \multirow[t]{3}{*}{15} & 10.5 & 18.1 & 51.9 & 69.5 & 109 & 151 & 197 \\
\hline & 10.5 & & & & & & 203 \\
\hline & 9.40 & & & & & & 196 \\
\hline \multirow[t]{3}{*}{16} & 10.2 & 17.4 & 54.6 & 71.1 & 108 & 138 & 193 \\
\hline & 10.5 & & & & & & 212 \\
\hline & 9.83 & & & & & & 200 \\
\hline Mean & 10.1 & 19.0 & 49.0 & 76.5 & 103 & 149 & 199 \\
\hline S.D. & 0.669 & 1.51 & 3.69 & 4.94 & 5.89 & 6.33 & 7.24 \\
\hline$\% \mathrm{CV}$ & 6.62 & 7.95 & 7.53 & 6.46 & 5.72 & 4.25 & 3.64 \\
\hline \%Bias & 1.00 & -5.00 & -2.00 & 2.00 & 3.00 & -0.67 & -0.50 \\
\hline $\mathrm{n}$ & 47 & 15 & 16 & 16 & 16 & 16 & 48 \\
\hline \multicolumn{8}{|l|}{ Reasons Deactivated } \\
\hline \multicolumn{8}{|l|}{${ }^{*}>25 \%$ of acceptance criteria, not in statistics } \\
\hline${ }^{* *}>20 \%$ of acceptance criteria, not in statistics & & & & & & & \\
\hline
\end{tabular}


Table S2. Calibration curve regression parameters for alemtuzumab.

\begin{tabular}{cccccccc}
\hline $\begin{array}{c}\text { Curve } \\
\text { Number }\end{array}$ & A & B & C & $\begin{array}{c}\text { R- } \\
\text { Squared }\end{array}$ & LLOQ & ULOQ & $\begin{array}{c}\text { Regression } \\
\text { Footnote(s) }\end{array}$ \\
\hline 1 & 13384 & 5188100 & -19709000 & $\mathbf{0 . 9 8 9 4}$ & 10.0 & 200 & 1 \\
2 & 16813 & 4312600 & -15217000 & $\mathbf{0 . 9 9 7 2}$ & 10.0 & 200 & 1 \\
3 & 11702 & 3479200 & -16997000 & $\mathbf{0 . 9 9 4 3}$ & 10.0 & 200 & 1 \\
4 & 11491 & 2850500 & -9746900 & $\mathbf{0 . 9 9 7 7}$ & 10.0 & 200 & 1 \\
5 & 10413 & 5827600 & -24477000 & $\mathbf{0 . 9 9 4 9}$ & 10.0 & 200 & 1 \\
6 & 5015.6 & 6166500 & -27880000 & $\mathbf{0 . 9 9 2 4}$ & 10.0 & 200 & 1 \\
7 & 17873 & 3973700 & -21794000 & $\mathbf{0 . 9 9 5 1}$ & 10.0 & 200 & 1 \\
8 & 16155 & 3244900 & -10893000 & $\mathbf{0 . 9 9 8 0}$ & 10.0 & 200 & 1 \\
9 & 10964 & 3468400 & -13533000 & $\mathbf{0 . 9 9 2 1}$ & 10.0 & 200 & 1 \\
10 & 18625 & 3245700 & -11347000 & $\mathbf{0 . 9 9 6 5}$ & 10.0 & 200 & 1 \\
11 & 14867 & 3824300 & -16492000 & $\mathbf{0 . 9 9 5 3}$ & 10.0 & 200 & 1 \\
12 & 4045.9 & 1897800 & -8877900 & $\mathbf{0 . 9 8 9 9}$ & 10.0 & 200 & 1 \\
13 & 9248.7 & 1849700 & -7174600 & $\mathbf{0 . 9 8 7 2}$ & 10.0 & 200 & 1 \\
14 & 4757.2 & 430880 & 6448900 & $\mathbf{0 . 9 9 4 4}$ & 10.0 & 200 & 1 \\
15 & 4967.7 & 1093500 & -7128200 & $\mathbf{0 . 9 9 5 4}$ & 10.0 & 200 & 1 \\
16 & 7367.0 & 1227900 & -8889200 & $\mathbf{0 . 9 9 0 9}$ & 10.0 & 200 & 1
\end{tabular}

Regression Footnote(s):

1) Resp. $=A$ * (Conc. $)^{2}+B *$ Conc. $+C$ 
Table S3. Intra- and inter-runs accuracy and precision of the assay performed using alemtuzumab on three separate occasions.

\begin{tabular}{|c|c|c|c|c|}
\hline $\begin{array}{c}\text { QC nominal conc. } \\
(\mu \mathrm{g} / \mathrm{mL})\end{array}$ & $\begin{array}{c}\text { QC mean calculated } \\
\text { conc. }(\mu \mathrm{g} / \mathrm{mL})\end{array}$ & $\begin{array}{c}\text { Accuracy } \\
\text { (Bias \%) }\end{array}$ & $\begin{array}{c}\text { Total precision } \\
\text { (CV\%) }\end{array}$ & $\begin{array}{c}\text { Number of } \\
\text { replicates }\end{array}$ \\
\hline $10.0(\mathrm{LLOQ})$ & 11.1 & $\mathbf{1 1 . 0 0}$ & $\mathbf{1 3 . 0 3}$ & 18 \\
\hline 30.0 & 29.7 & $\mathbf{- 1 . 0 0}$ & $\mathbf{1 1 . 2 3}$ & 18 \\
\hline 100 & 91.1 & $\mathbf{- 8 . 9 0}$ & $\mathbf{6 . 5 5}$ & 18 \\
\hline 180 & 165 & $\mathbf{- 8 . 3 3}$ & $\mathbf{9 . 9 6}$ & 18 \\
\hline
\end{tabular}


Table S4. Influence of dilution on the quantification of alemtuzumab $(\mu \mathrm{g} / \mathrm{mL})$ in human serum.

\begin{tabular}{|c|c|c|c|}
\hline Nominal & $\begin{array}{l}\text { Dilution factor } 10 \\
1000 \mu \mathrm{g} / \mathrm{mL}\end{array}$ & $\begin{array}{c}\text { Dilution factor } 20 \\
1000 \mu \mathrm{g} / \mathrm{mL}\end{array}$ & $\begin{array}{c}\text { Dilution factor } 50 \\
1000 \mu \mathrm{g} / \mathrm{mL}\end{array}$ \\
\hline & 984 & 820 & 827 \\
\hline & 871 & 923 & 913 \\
\hline \multirow[t]{4}{*}{ Observed } & 961 & 903 & 900 \\
\hline & 859 & 910 & 875 \\
\hline & 830 & 1010 & 849 \\
\hline & 990 & 873 & 901 \\
\hline Mean & 916 & 907 & 878 \\
\hline S.D. & 70.4 & 62.6 & 33.7 \\
\hline$\% \mathrm{CV}$ & 7.69 & 6.90 & 3.84 \\
\hline$\%$ Bias & -8.40 & -9.30 & -12.20 \\
\hline $\mathrm{n}$ & 6 & 6 & 6 \\
\hline
\end{tabular}


Table S5. Influence of matrix variability on quantitation of commercial M-protein (IgG1 kappa from Sigma at $20.4 \mu \mathrm{g} / \mathrm{mL}$ ) in 6 different batches of serum from healthy individuals (concentrations expressed as $\mu \mathrm{g} / \mathrm{mL}$ alemtuzumab equivalent).

\begin{tabular}{|c|c|c|c|c|c|c|}
\hline & Batch 1 & Batch 2 & Batch 3 & Batch 4 & Batch 5 & Batch 6 \\
\hline Mean conc. & 21.1 & 21.8 & 21.5 & 21.5 & 21.7 & 20.4 \\
\hline \% CV & $\mathbf{2 . 8 6}$ & $\mathbf{1 2 . 4 8}$ & $\mathbf{4 . 1 3}$ & $\mathbf{1 . 4 9}$ & $\mathbf{1 4 . 7 9}$ & $\mathbf{7 . 6 0}$ \\
\hline \% Bias & $\mathbf{3 . 4 3}$ & $\mathbf{6 . 8 6}$ & $\mathbf{5 . 3 9}$ & $\mathbf{5 . 3 9}$ & $\mathbf{6 . 3 7}$ & $\mathbf{0 . 0 0}$ \\
\hline Replicates & 3 & 3 & 3 & 3 & 3 & 3 \\
\hline
\end{tabular}


Table S6. M-protein LC deconvoluted mass for the patient with VGPR (Fig. 3) at each treatment cycle showing the high precision and robustness of the method.

\begin{tabular}{|c|c|}
\hline Treatment cycle & M-protein LC Deconvoluted mass (Da) \\
\hline Pretreatment & 23121.3 \\
\hline 8 & 23121.2 \\
\hline 9 & 23121.3 \\
\hline 10 & 23121.2 \\
\hline 11 & 23121.2 \\
\hline 12 & 23121.2 \\
\hline
\end{tabular}

\section{AUTHOR INFORMATION}

\section{Corresponding author:}

Phone: +33 4997747 85. E-mail: stephane.muccio@sanofi.com Notes:

All co-authors are Sanofi's employees. 